\title{
Population and spatial dynamics mangrove jellyfish Cassiopeia sp at Kenya's Gazi bay
}

\author{
Tsingalia H. M. \\ Department of Biological Sciences, Moi University, Box 3900-30100, Eldoret, Kenya
}

Email address:

mugatsi2005@mu.ac.ke

\section{To cite this article:}

Tsingalia H. M.. Population and Spatial Dynamics Mangrove Jellyfish Cassiopeia sp at Kenya's Gazi Bay. American Journal of Life Sciences. Vol. 2, No. 6, 2014, pp. 395-399. doi: 10.11648/j.ajls.20140206.20

\begin{abstract}
Cassiopeia, the upside-down or mangrove jellyfish is a bottom-dwelling, shallow water marine sycophozoan of the phylum Cnidaria. It is commonly referred to as jellyfish because of its jelly like appearance. The medusa is the dominant phase in its life history. They have a radial symmetry and occur in shallow, tropical lagoons, mangrove swamps and sandy mud falls in tropical and temperate regions. In coastal Kenya, they are found only in one specific location in the Gazi Bay of the south coast. There are no documented studies on this species in Kenya. The objective of this study was to quantify the spatial and size-class distribution, and recruitment of Cassiopeia at the Gazi Bay. Ten 50mx50m quadrats were randomly placed in an estimated study area of 6.4ha to cover about 40 percent of the total study area. A total of 1043 individual upside-down jellyfish were sampled. In each quadrat, all jellyfish encountered were sampled individually. For each individual jellyfish, the diameter was determined using a standard ruler. The colour and number of tentacles on each individual was determined and its activity at the time, recorded. Because jellyfish occurred in tidal pools, the number of individuals in each pool was determined. The percentage vegetation cover in each pool was estimated and recorded to the nearest whole number. There were predominantly more light brown individuals followed by dark brown with the least number of individuals being grey. Majority of the individuals were feeding. Most individuals had a mean diameter of 8.0-8.9 $\mathrm{cm}$. Individuals were well represented in the sizeclasses $4.0-4.9 \mathrm{~cm} ; 5.0-5.9 ; 6.0-6.9 \mathrm{~cm}$ and $7.0-7.9 \mathrm{~cm}$. Very few individuals occurred in the $12.0-12.9 \mathrm{~cm}$ class. More individuals were found in clumps than singly more so where the sea grass dominated. If the diameter of the jellyfish correlates with the age, then the population structure of the jellyfish at Gazi Bay is that of a growing population. Diameter appears to have been the single most factors that influenced spacing. The smaller the individuals, the more likely they were to be found in clumps compared to larger individuals. It is possible that clumping was also related to the size of the tidal pools. This study is significant in being the first study to document the population biology of the jellyfish at the Kenyan coast. Future studies are recommended to determine factors that restrict the distribution of this species to a specific location at the south coast of Kenya. The presence of Cassiopeia at the Gazi lagoon adds value to tourism attraction of the area.
\end{abstract}

Keywords: Upside-Down Jellyfish, Cassiopeia, Population Structure, Spatial Dynamics, Size-Class Distribution, Marine, Gazi Bay

\section{Introduction}

Cassiopea $\mathrm{sp}$ is a large jellyfish that belongs to the phylum Cnidaria and class scyphozoan. It is commonly referred to as the upside-down or mangrove jellyfish (Fitt and Costley 1998; Kaplan 1998; Holland et al. 2004). Its name is derived from the fact that it tends to stay upside down in shallow, clear waters that it inhabits. The upside down jellyfish is also known as the mangrove jellyfish because it mainly inhabits the mangroves in the Caribbean and southern Gulf of Mexico among other tropical waters where the genus has been well- studied (Fleck and Fitt 1999; Hofmann and Hadfield 2002; Muller and Leitz 2002). This species has also been reported in muddy marine areas by Kaplan (1988).

Cassiopeia is a widely distributed genus. It has been reported in tropical Pacific and western Atlantic, Bermuda and south Florida which marks the northern limit of the range of the Caribbean Cassiopeia species (Sterrer 1992; Fleck and Fitt 1999). The upside down jellyfish differs from other jellyfishes by looking less like a jellyfish and more like a sea anemone. It can be easily mistaken for a bluish green water flower. However, this appearance of the upside down jellyfish is very important for its survival, for it provides 
excellent good camouflage against its predators. Apart from its appearance, the upside down jellyfish is unique in another important aspect. Instead of just hunting its own food, the upside down jellyfish depends upon unicellular algae, called zooxanthellae, for partial nutrition. The algae use photosynthesis to create nutrition from sunlight. The nutrition created by the algae is absorbed by the upside down jellyfish for its own survival. This symbiotic relationship between the upside down jellyfish and the algae has been proposed as the reason for the upside down nature of the jellyfish (Murphy, 2002). The algae reside inside the bell of the jellyfish and need sunlight to photosynthesize food. To enable the algae to access sunlight, the jellyfish floats upside down in water. This also explains why the upside down jellyfish resides in shallow water. That way they can settle upside down on the bed of the water body, while providing ample sunlight to the algae inside its bell. The upside down jellyfish also makes use of its stinging cells, the nematocysts, to capture its primary source of nutrition, mainly plankton and zooplankton. Floating plankton and zooplankton get paralyzed when they come in contact with the nematocysts and become food for the upside down jellyfish. Once the prey has been paralyzed, the tentacles also help transport the food to the mouth of the upside down jellyfish. There is another interesting aspect to the upside down jellyfish. Instead of having a single primary mouth, the upside down jellyfish has a primary mouth and a series of secondary mouths (Cooke, 1984). The primary mouth is used to break the food down into small particles, which are then ingested by the secondary mouths. The upside down jellyfish has a number of other fascinating relationships. One interesting relationship of the upside down jellyfish is that is a regularly used by water crabs as a form of protection. When these crabs reach the surface or the edge of the waterbed, they tend to carry upside down jellyfish on their backs. They do so to protect themselves from their likely predator by using the tentacles of the upside down jellyfish as their shield. Jellyfish are important organisms and play important ecological roles. Their life spans a period of between 3-6 months. They exist in two body forms - the polyp and medusoid forms that ensure both sexual and asexual reproduction. They use their tentacles as defense organs. They are covered with cnidocytes which are stinging cells that are used to inject poison into their prey. They move by generating a water current using their tentacles and pulsating swimming tendencies in search for food.

This study examined the population structure and spatial dynamics of the upside down jellyfish and the factors that influences these characteristics. This study was important because there is little documentation of this species at the Kenya's coast. Information of the population structure is important because it will shed light on the status of the population that is found restricted to a very specific area of Kenya's south coast.

\section{The Study Area}

This study was conducted at the Gazi Bay at Kenya's south coast at $04025.631 \mathrm{~S}, 039030.929 \mathrm{E}$. It is located some $20 \mathrm{~km}$ from the Ukunda town but some $60 \mathrm{~km}$ from Mombasa town. This area is $12 \mathrm{~m}$ above sea level and covers an estimated area of $0.062 \mathrm{~km} 2$. The Gazi Bay is a shallow sub tidal mud and sand flats that are sheltered by fringing coral reefs. Twelve species of sea grass grow on the mud flats and the sea grass beds cover about half of the bay's $15 \mathrm{~km} 2$. Mangrove lined creeks flow into the bay and this unusual proximity of the mangrove, sea grass and coral reef systems has stimulated a lot of scientific interests in their interaction. The sea grass function as important trappers of particles washed into the bay form the creeks. The sea grass beds also provide food directly for other marine intertidal organisms such larvae shrimps, zooplankton and oysters and are the main feeding ground for all fish in the bay making them useful to the health of the local fisheries. Within the study area were many isolated tidal black muddy pools of varied sizes. It is inside and around these pools that jellyfishes were concentrated. The study area was also characterized by a dense cover of sea grass in which many individuals of the jellyfish were found.

\section{Materials and Methods}

The quadrat method was used to sample the study area. $50 \mathrm{mx} 50 \mathrm{~m}$ quadrats were randomly sited $100 \mathrm{~m}$ apart in study area measuring $150 \mathrm{~m} \times 500 \mathrm{~m}$. The actual area was plotted using Geographical Positioning System (GPS). A total of 10 quadrats were sampled. In each quadrat, all individual jellyfishes were counted. For every individual encountered, its colour, diameter, number in each tidal pool and the percentage cover by sea grass were determined. Percentage vegetation cover for each tidal pool was roughly estimated by comparing the total area of the quadrat with that covered by vegetation which was mainly sea grass. Vegetation cover was estimated to the nearest whole number. The number of tentacles on each individual encountered was determined. Air and water temperatures were determined at the beginning and at the end of the exercise. Salinity of the water at the beginning and end of the sampling regime was also determined by titration using silver nitrate solution.

\section{Results and Discussion}

\subsection{Population Structure and Activity of the Jellyfish}

The total jellyfish habitat at the Gazi bay was estimated at 6.4ha of which 2.5 ha were sampled through the quadrat method representing some 40 per cent of the total area. A total of 1043 individual jellyfish were sampled. Using extrapolation, it was estimated that there were slightly more than 2600 individuals of jellyfish in the entire study area. Most of the jellyfish had eight (8) tentacles $(90.8 \%$; $n=946)$ while very few had seven (7) tentacles $(8.2 \% ; n=83)$. Majority of the jellyfish were actively feeding (93.7\%; $n=977)$ and only an insignificant number were dormant $(6.3 \% ; n=66)$.

An interesting feature of the jellyfish was the existence of differently coloured individuals (coloured morphs). A close 
scrutiny of the proportions of the different individuals revealed that there were three types of coloured jellyfish. There were Light brown, dark brown and grey coloured individuals. Interestingly, the different morphs were not spatially separated. The light brown morph was more frequent 55.4 percent $(n=576)$ reflecting their adaptive success compared to the other two morphs. The light brown colour morphs comprised 35.3 per cent $(n=368)$ while the grey coloured morphs comprised 9.3 percent $(n=97)$. The colour morphs were significantly different (ANOVA, $\mathrm{F}=2.604 ; \mathrm{p}<0.001)$.

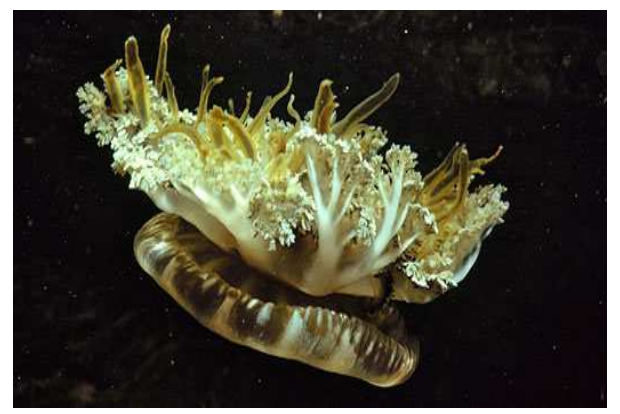

a

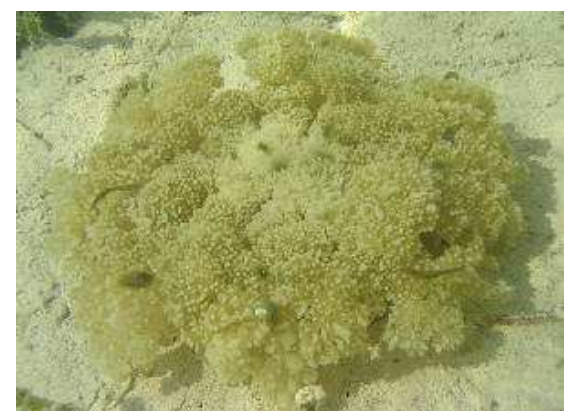

b

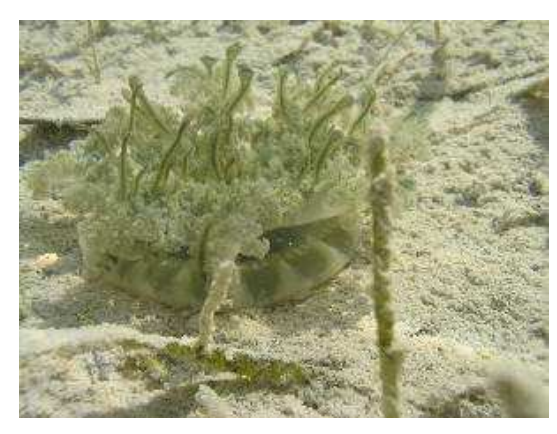

Figure 1. Different types of Coloured jellyfish - (a) dark brown, (b) light brown and (c) grey respectively.

It is not clear which selective pressures favour the light brown individuals and further studies are required. It is also not clear whether the different morphs interbreed which would account for the differences in colouration.

\subsection{Spatial and Size Class Distribution}

Figure 2 shows the size class distribution by diameter of the Gazi jellyfish population. The population adheres to the normal distribution curve suggesting a stable population. The mean diameter for individual jellyfish was $7.68 \mathrm{~cm}$. Majority of the individual were in the size class 5-9 with very few individual in lower and higher diameter size-classes. The largest individuals were in the diameter size-class $15 \mathrm{~cm}$.

Majority of the jellyfish individuals at the Gazi Bay study site were patchily distributed. The patchy distribution was dependent on the tidal pools at low tide. To ascertain the determinants of clump size, a partial correlation was done for the diameter of the individuals, colour and percentage vegetation with the number in the clump being the control. The diameter of the individual significantly accounted for clump size $(\mathrm{p}<0.0001,2$-tailed $)$ while the colour of the individuals did not ( $\mathrm{p}=0.472$, 2-tailed; fig. 3). Clumping was evident in individual of $2-3 \mathrm{~cm}$ diameter size class. It clears that smaller individuals have a higher tendency to clump probably for feeding efficiency. Larger individuals were least likely to be found in clumps. Vegetation cover was also correlated with clumping (Pearson correlation=0.753, $\mathrm{p}<0.001$, 1-tailed). Figure 3 shows the clumping of individuals by diameter class size.

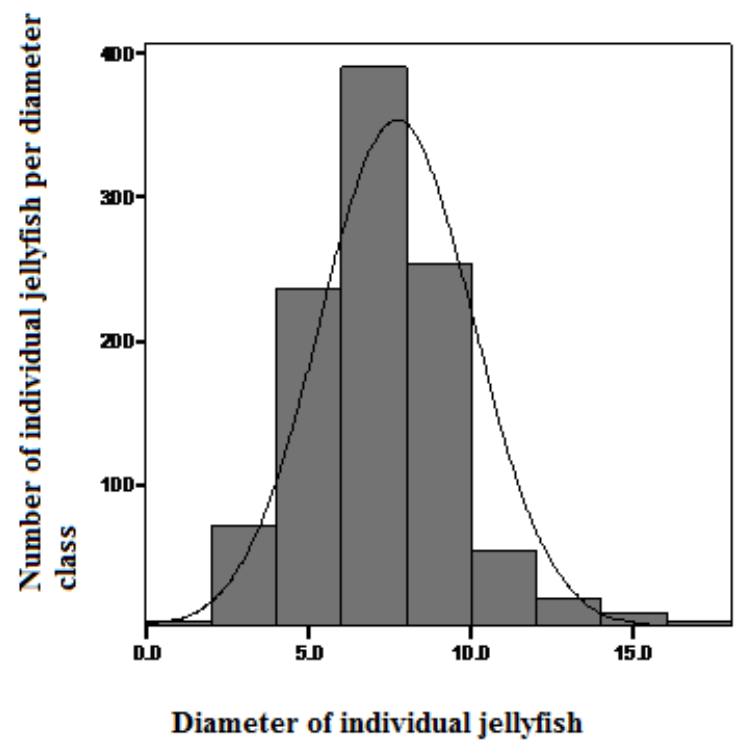

Figure 2. Size class distribution by diameter of individual jellyfish.

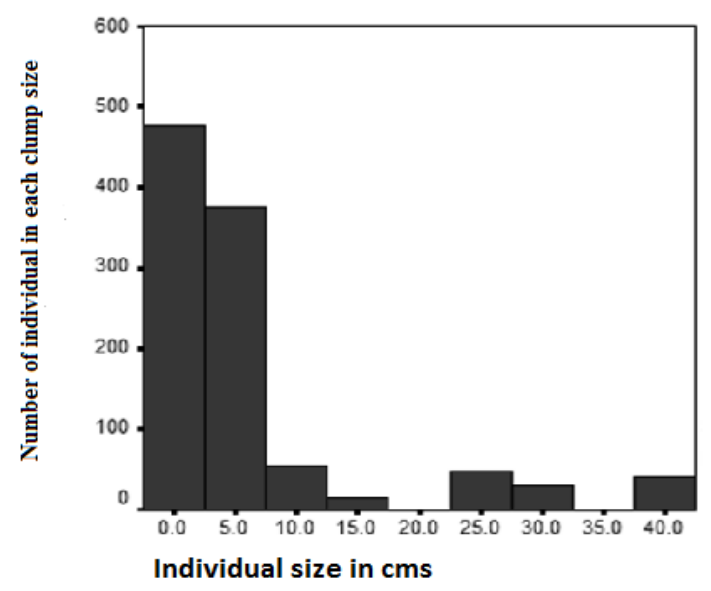

(a) 


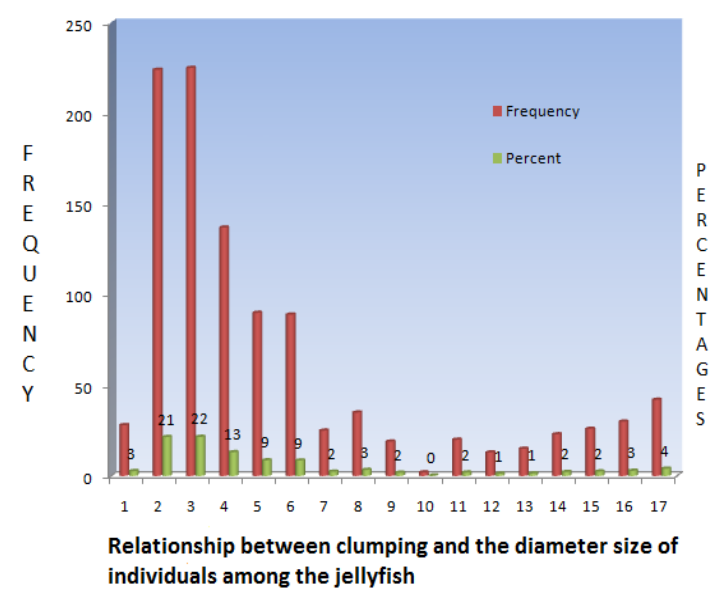

(b)

Figure 3. (a) Clumping as a function of the size of individuals. Many individuals in the large diameter classes were not clumped. (b) Clumping as a function of diameter class size.

Clumping was also influenced by the colour of the jellyfish and appeared to vary with the colour morph of individuals (figure 4). Clumping was strongly correlated with colour of the individual (Spearman rho $=0.103, \mathrm{p}<0.001,1$ tailed). The light brown colour morph exhibited higher clumping than the grey and dark-brown colour morphs. The grey colour morph exhibited the least clumping in diameter class. The dark brown colour morph exhibited maximum clumping in diameter class 3 while the clumping in light brown morph was by individuals of $2 \mathrm{~cm}$ diameter. The differences in the tendency to clump appear to be additionally influenced by the colour morph.

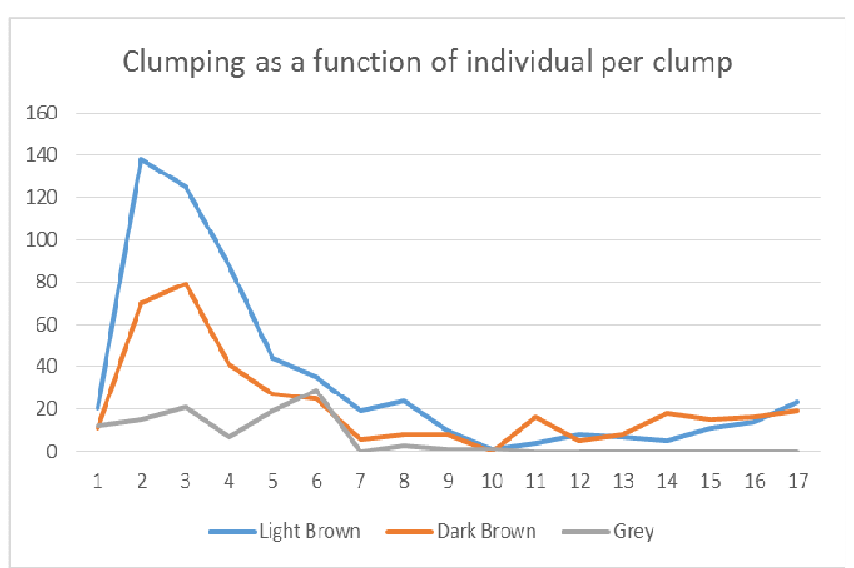

Figure 4. Differences in clumping by colour of jellyfish.

Only 14.6 per cent $(n=152)$ of the jellyfish were found in tidal pools that were devoid of vegetation. Majority of the individual were in tidal pools with vegetation cover of varying percentages. When percent vegetation cover was classified, then close to half of the sampled jellyfish were in pools with a vegetation cover of $\leq 15$ per cent (fig. 5). This may be explained by the symbiotic relationship between jellyfish and green algae which require sunlight to photosynthesize. Slightly less than 300 individuals occurred in tidal pools with a vegetation cover less than 50 per cent further reinforcing the need for sunlight by jellyfish.

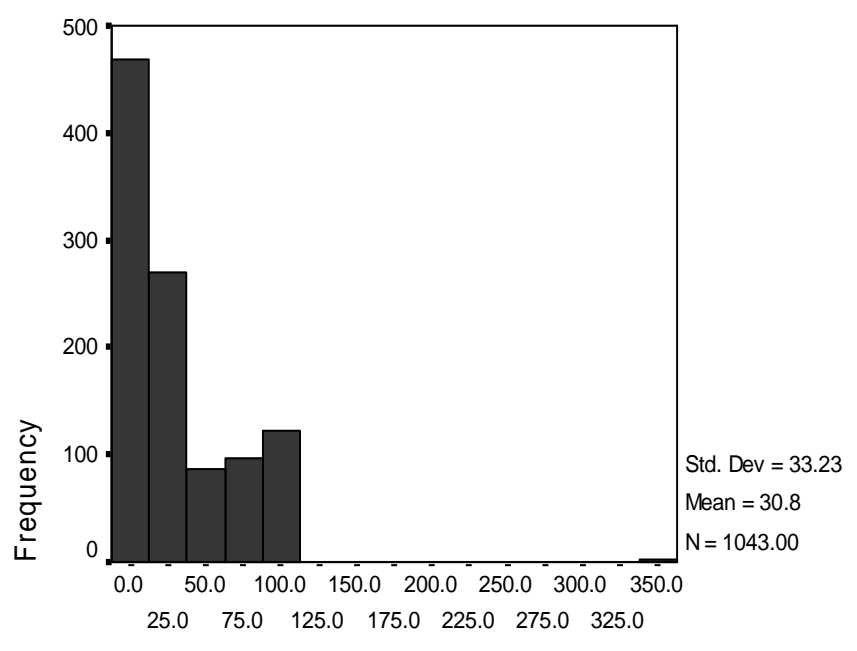

Percenatge vegetation cover of pools

Figure 5. Percentage vegetation cover of different tidal pools.

\section{Conclusions and Recommendations}

Results at the time of sampling clearly show that the population of the Cassiopeia sp. is stable with representatives in all diameter classes. This, however, needs to be supported by more long-term data on size-class distribution over slightly longer periods. This species is found in a very restricted area in the Gazi bay and whereas studies have found individuals as large as $30 \mathrm{~cm}$ the largest species in this study was $15 \mathrm{~cm}$. Further studies need to be done to identify sites along the Kenyan coast that may contain this species and how the populations differ from site to site. The tendency to show clumping is strongly correlated with diameter size. The clumping among smaller jellyfish may be a protective mechanism that requires further investigation.

An interesting feature of this species is the existence of colour morphs that are significantly different. This has not been reported before. Colour morph has significant effect on clumped distribution. Further studies are required to determine the evolutionary significance of these colour morphs.

\section{References}

[1] Cooke, W.J. 1984. New scyphozoan records for Hawaii: Anomalorhiza shawi Light 1921 and Thysanostoma loriferum (Ehrenberg 1835); with notes on several other rhizostomes. Proc. Biol. Soc. Wash. 97: 583-588.

[2] Fitt, W.K., 1984. The role of chemosensory behavior of Symbiodinium microadriaticum, intermediate hosts, and host behavior in the infection of coelenterates and mollusks with zooxanthellae. Mar. Biol. 81: 9-17.

[3] Fitt, W.K., Costley, K., 1998. The role of temperature in survival of the polyp stage of the tropical rhizostome jellyfish Cassiopea xamachana. J. Exp. Mar. Biol. Ecol. 222: 79-91. 
[4] Fleck, J., Fitt, W.K., 1999. Degrading mangrove leaves of Rhizophora mangle Linne provide a natural cue for settlement and metamorphosis of the upside down jellyfish Cassiopea xamachana Bigelow. J. Exp. Mar. Biol. Ecol. 234: 83-94.

[5] Hoffman, D.K., Neumann, R., Henne, K., 1978. Strobilation, budding and initiation of scyphistoma morphogenesis in the rhizostome Cassiopea andromeda. Mar. Biol. 47: 161-176.

[6] Hoffman, D.K., Fitt, W.K., Fleck, J., 1996. Checkpoints in the life-cycle of Cassiopea spp.: control of metagenesis and metamorphosis in a tropical jellyfish. Int. J. Dev. Biol. 40: 331-338.

[7] Müller, W.A., Leitz, T., 2002. Metamorphosis in the Cnidaria. Can. J. Zool. 80: 1755-1771.

[8] Murphy, R.C. 2002. Coral Reefs: Cities under the Sea. Princeton, New Jersey: The Darwin Press. ISBN 978-087850-138-0.

[9] Niggle W, Naumann MS, Struck U, Manasrah R, Wild C. 2010. Organic matterrelease by the benthic upside-down jellyfish Cassiopea sp. fuels pelagic food webs in coral reefs. J. Exp. Mar.Biol. and Ecol., Vol., 384 (1\&2), pages 99-106.

[10] Passano, L.M., 2004. Spasm behavior and the diffuse nervenet in Cassiopeaxamachana (Scyphozoa: Coelenterata). Hydrobiologia, 530-531(1): 91-96.

[11] Radwan, F.F.Y., Roman, L.G., Baksi, K., Burnett, J.W., 2005. Toxicity and mAChRs binding activity of Cassiopea xamachana venom from Puerto Rican coasts. Toxicon 45(1): 107-112.
[12] Rahat, M., Adar, O., 1980. Effect of symbiotic zooxanthellae and temperature on budding and strobilation in Cassiopea andromeda. Biol. Bull. 159: 394-401.

[13] Ruppert, E.E., Fox, R.S., Barnes, R.D., 2004. Invertebrate zoology. A functional evolutionary approach. Thomson, Brooks/Cole, USA. pp. 148-153.

[14] Sterrer, W., 1986. Marine fauna and flora of Bermuda. A systematic guide to the identification of marine organisms. John Wiley \& Sons, Inc. pp. 158-159.

[15] Sterrer, W., 1992. Bermuda's marine life. Bda. Zool. Soc., Island Press, Bda. pp. 43-44.

[16] Vodenichar, J.S., 1995. Ecological physiology of the scyphozoan Cassiopea xamachana. M.S. Thesis, University of Georgia, Athens, USA.

[17] Wood, J.B., Jackson, K.J., 2005. Bermuda. In: Caribbean marine biodiversity: the known and the unknown. Miloslavich, P. \& Klein, E., Eds. DEStech Publications, Inc., Lancaster, PA. pp. 19-35.

[18] http://www.ncbi.nlm.nih.gov/Taxonomy/Browser/wwwtax.cgi ?mode $=$ Info\&id $=37536 \& l v l=3 \&$ lin $=f \& k e e p=1 \&$ srchmode $=1$ \&unl

[19] http://www.jellyfishfacts.net/news/mangrove-jellyfishcassiopea-xamachana-nid-78.html\#ixzz1QRqZXeV2

[20] http://blogs.thatpetplace.com/thatfishblog/2009/01/12/clearing -cloudy-water-common-aquarium-questions

[21] http://www.jellyfishfacts.net/upside-downjellyfish.html\#ixzz1QRgD4YX0 